\title{
Management of elderly and unfit patients with chronic lymphocytic leukemia
}

\author{
Francesca R. Mauro, Adriano Salaroli, Maria D. Caputo, Gioia Colafigli, Luigi \\ Petrucci, Melissa Campanelli, Antonietta Ferretti, Anna R Guarini \& Robin \\ Foà
}

To cite this article: Francesca R. Mauro, Adriano Salaroli, Maria D. Caputo, Gioia Colafigli, Luigi Petrucci, Melissa Campanelli, Antonietta Ferretti, Anna R Guarini \& Robin Foà (2016): Management of elderly and unfit patients with chronic lymphocytic leukemia, Expert Review of Hematology, DOI: 10.1080/17474086.2016.1254544

To link to this article: http://dx.doi.org/10.1080/17474086.2016.1254544

Accepted author version posted online: 31 Oct 2016.

Submit your article to this journal

a)

View related articles $\widetilde{ }$

View Crossmark data $₫$ 
Publisher: Taylor \& Francis

Journal: Expert Review of Hematology

DOI: $10.1080 / 17474086.2016 .1254544$

Review

Management of elderly and unfit patients with chronic lymphocytic

\section{leukemia}

Francesca R. Mauro, Adriano Salaroli, Maria D. Caputo, Gioia Colafigli, Luigi Petrucci, Melissa Campanelli, Antonietta Ferretti, Anna R Guarini, Robin Foà

Hematology, Department of Cellular Biotechnologies and Hematology, Policlinico Umberto 1 and Sapienza University, Rome, Italy.

\section{CORRESPONDING AUTHOR:}

Francesca R Mauro, Hematology, Department of Cellular Biotechnologies and Hematology, Sapienza University, Via Benevento 6, 00161 Rome, Italy.

E-mail: mauro@bce.uniroma1.it

Phone: +3906 499741

Fax: +390644241984 


\begin{abstract}
Introduction: About $75 \%$ of patients with chronic lymphocytic leukemia (CLL) are more than 65 years at the time of diagnosis. Treatment of the elderly remains complicated due to multiple factors, such as comorbidities, decline in functional reserve and fitness. Since chronological age by itself cannot properly predict life expectancy and treatment tolerance, an accurate assessment of the fitness status is of crucial importance for an optimal treatment choice.
\end{abstract}

Areas covered: This review will discuss the most relevant aspects concerning the issues experienced in the management of elderly/unfit patients with CLL. The most frequently observed age-related toxicities, fitness assessments, supportive care measures and treatment options for elderly patients and for patients who are deemed unfit will be discussed. Literature search methodology included examination of PubMed index.

Expert commentary: During the last decade, different trials focusing on elderly/unfit patients have investigated more tolerable chemoimmunotherapy schedules and, more recently, the activity and safety of chemo-free regimens. Chlorambucil combined with an anti-CD20 monoclonal antibody has shown clinical activity with a relatively good profile of toxicity. The recent introduction of the Bcell receptor antagonists, ibrutinib and idelalisib, and other targeted drugs in development (e.g., venetoclax), is broadening the therapeutic armamentarium of elderly CLL patients.

\title{
KEYWORDS
}

Chronic lymphocytic leukemia, older, elderly, comorbidities, front-line treatment 


\section{INTRODUCTION}

\subsection{Epidemiology of CLL}

Chronic lymphocytic leukemia (CLL) is the most frequent leukemia diagnosed among adults in the western world. According to the 2014 statistics from the National Cancer Institute in the U.S., the median age of CLL patients at diagnosis is around 70 years (1).In some studies patients over this age were defined as older (2) while in others, over 65 years has been considered as the age limit to define older patients (3-5). As life expectancy is increasing, many countries are experiencing an increase in older individuals and by the year 2030 people over $65-70$ years are expected to account for more than $70 \%$ of all patients with cancer. On this basis, the prevalence of CLL is expected to grow over the next few years due to the increase of elderly people.

Fludarabine-based regimens have improved significantly the response and outcome of CLL patients [6-8]. However, elderly/unfit patients tolerate poorly these regimens because of comorbidities that increase after the age of 70 [4,9]. Bendamustine and chlorambucil- based regimens are better tolerated by older patients but are associated with a lower response duration. This finding can explain why the improvement in the outcome of CLL patients recorded over the past several decades does not include older patients [10].

\subsection{The effect of age in the outcome of CLL patients}

Advanced age is by itself a high risk factor for cancer. The majority of studies report age as an independent adverse factor for the survival of CLL patients [2, 11-15]. However, it should be noted that CLL, and no other age-related diseases, is the most frequently cause of death for the majority of older patients. The study by Shanafelt et al. [13] demonstrates clearly that CLL is a mortality risk 
even in patients with Rai stage 0 who show a lower survival probability than that observed in subjects of the same age. There are no data supporting that an adverse biologic profile is more frequent in older than in younger patients.

Bauman et al. [2] investigated the clinico-biological features, outcomes, and prognosis of 949 patients with CLL according to age. Elderly patients ( $>70$ years; $n=367$ ) did not show significant biological differences when compared to younger patients. Interestingly, in several studies, age emerged as an adverse prognostic factor independent from the heavy chain variable IGVH mutational status or the presence of poor prognosis cytogenetic aberrations $[12,14,15]$. In a retrospective study the poor outcome of elderly patients was associated with the presence of comorbidities representing an obstacle to the administration of an adequate treatment [2]. Elderly patients were treated less frequently than younger patients and in most cases, did not receive highly effective regimens. This translated into a significantly lower rate of complete responses and in a shorter survival. An older age was associated with a significantly higher disease-unrelated mortality. However, disease-attributable mortality was not significantly different between younger and older patients.

The need of more appropriate treatments for aged patients has led to investigate specific regimens for unfit and/or elderly patients. In this setting, different trials have explored the benefit in unfit/elderly patients of schedules including chlorambucil associated with an anti-CD20 monoclonal antibody [17-20], lenalidomide [5, 21] and more recently, ibrutinib [22-23] and idelalisib combined with rituximab $[24,25]$.

\section{ASSESSMENT OF ELDERLY PATIENTS WITH CLL}

\subsection{Patients' expectations}

While for younger and fit patients, a more aggressive treatment aimed at achieve MRD negative complete response, may be considered the optimal treatment approach, prolonging survival in older patients should be attempted without occurring in unnecessary treatment-related toxicity. In this prospective, when making a treatment decision, the benefits and the risks of early and longterm toxicities should be considered and clearly discussed with patients. 


\subsection{Age and treatment-related toxicity}

Aging is associated with a more pronounced myelotoxicity and susceptibility to infections. The immunosenescence, the state of dysregulated immune function associated with increasing age, play an important contributory role towards the risk of infections. Emerging data suggest that progressive aging significantly affects frequencies, subset distribution and functional competence of regulatory $\mathrm{T}$ cells [26]. In addition, chemotherapy-induced neutropenia is more frequent in elderly and significantly increases the risk of life-threatening infectious complications [27].

At present, there is limited information with regard to the effects of age on the toxicity of kinase inhibitors. It can be expected that some adverse events - atrial fibrillation (AF), hypertension, intestinal disorders, cytopenias - could be more frequently observed in older than in younger patients receiving BCR antagonists.

\subsection{Prognostic effect of comorbidities}

The decline in functional organ reserve combined with an increase in the number of comorbidities associated with aging, is highly variable and this is why chronological age by itself cannot predict properly the life expectancy of individual patients as well as the treatment tolerance.

The negative prognostic impact of comorbidities has been described in large series of patients with different cancers [28]. In a Mayo Clinic study including 373 unselected CLL patients the presence of one or more comorbidities was recorded in $89 \%$ of patients, and $46 \%$ had at least one major comorbidity [29]. Major comorbidities revealed an adverse impact on survival in univariate analysis but lost their significance in multivariate analysis when disease-related variables were tested. This observation suggests that disease-related factors had a prevalent effect on survival in elderly patients with CLL. Goede et al. investigated the impact of comorbidities in 555 patients with CLL enrolled in two trials of the German CLL Study Group (GCLLSG) [9]. Patients with two or more comorbidities showed a significantly lower overall survival (OS) and progression-free survival (PFS) than those with no or less comorbidities. However, in this study also CLL was the major cause of death in patients with two or more comorbidities. 
Similarly, data from a study based on the Surveillance, Epidemiology, and End Results (SEER) and Medicare database including 2.985 patients with CLL, showed that older age and an increasing comorbidity score were significantly associated with higher mortality. However, treatment including rituximab and intravenous chemotherapy was associated with a significantly lower mortality risk than treatments with intravenous chemotherapy only [1].

The adverse prognostic effect of comorbidities also emerged in the study by Bauman et al.[2]. In patients requiring treatment, comorbidities and response to treatment were the most important prognostic factors for survival.

Taken together, these observations indicate that a durable control of CLL is the most critical objective to reach in order to improve the outcome of fit, as well unfit patients. This observation underlines the importance of effective and tolerable treatment options for elderly/unfit patients.

\subsection{Assessment of the fitness status and comorbidities}

Several scoring systems, such as the CIRS score and the Charlson Comorbidity Index [30,31], are widely used for categorizing the severity of comorbidities in clinical trials. In particular, the CIRS scoring system is increasingly used in clinical trials to assess the comorbidity burden of CLL patients [31] (Table 1). The National Comprehensive Cancer Network guidelines [32] recommend that a geriatric assessment of patients - including function, comorbidity, polypharmacy, cognitive and emotional status, social support, nutrition and financial resources - should be considered in the pre-treatment work-up of older patients in order to estimate correctly the physiologic age and to define the most appropriate treatment approach.

A Spanish study carried out on older patients ( $\geq 70$ years) developed a prognostic score including CIRS scoring system ( $\leq 6$ versus $>6$ ) assessed at diagnosis, Binet stage, beta-2microglobulin, and ZAP-70, that discriminated CLL patients into two risk groups with significant differences in survival $[2]$.

However, despite the prognostic effect of comorbidities the CIRS scoring system has not been included in the prognostic scores that have been evaluated in newly diagnosed patients with CLL [33-36]. 


\section{MANAGEMENT OF TREATMENT-RELATED TOXICITIES AND SUPPORTIVE TREATMENT}

The progressive reduction in the functional reserve of multiple organ systems affects both pharmacokinetics and pharmacodynamics of chemotherapy leading to an increased risk of treatment complications. Furthermore, the outcome of coexisting chronic diseases may worsen toxicity $[6,37]$.

The effect of the reduction of the reserve of hemopoietic progenitors with age may be more relevant in CLL which is typically characterized by a profound bone marrow infiltration. The bone marrow involvement may explain the more pronounced chemotherapy-related cytopenias reported in elderly patients with CLL included in clinical trials. Neutropenic infections are associated with a considerable risk of prolonged hospitalization and mortality representing a relevant issue in the clinical management of older patients. Severe granulocytopenia is recorded in a variable proportion of older patients with CLL treated with chlorambucil given as a single agent or combined with a monoclonal antibody [17-20]. Trials focused on older patients treated with kinase inhibitors were also complicated by granulocytopenia (Table 2) [22-25].

Considering granulocytopenia, the CLL-related and treatment-related immune-defects, the physiological immunosenescence, a primary prophylaxis of granulocytopenia with myelopoietic growth factors should be considered in elderly patients [38-40]. Moreover, in the presence of symptomatic hypogammaglobinemia a support with immunoglobulins should be introduced. However, it should be noted that this recommendation is based on experts' opinion only.

While vaccines that contain live viruses, such as the shingles vaccine (herpes zoster vaccine) should be avoided, influenza and pneumococcal vaccinations may effectively mitigate the infection risk [41]. 
It is well known that the incidence and prevalence of anemia increases with age. Iron, vitamin B12 deficiency, inadequate levels of response to erythropoietin, myelodysplasia, other chronic diseases or malignancies associated with anemia should be investigated and managed [42]. The presence of anemia can worsen the fatigue usually marked in older patients and has by itself an adverse impact on the outcome of cardiac and neurological comorbidities. Vitamin D insufficiency is frequently observed in postmenopausal women and should be checked in female patients with CLL. Interestingly, vitamin D insufficiency is associated not only with osteoporosis and an increased risk of solid tumors but also with an inferior outcome of CLL. Two studies showed that Vitamin D insufficiency was associated with inferior time to treatment [43-44]. However, the benefit of vitamin repletion in CLL patients with vitamin D insufficiency has not been evaluated in controlled studies.

Among the elderly population, as well as in cancer patients, malnutrition is common and associated with an adverse outcome. The introduction of an adequate diet and vitamin supplement may improve treatment tolerance.

In addition, a high prevalence of depressive disorders is also frequently observed in cancer patients and in elderly people. In more aged patients with mobility limitations, cognitive symptoms, memory loss or other disabilities, a safe and successful treatment of CLL requires necessarily the presence of a well-trained familial or paid caregiver. The caregiver is essential not only to help with activities of daily living but also in the correct understanding and administering of medications and in the management of medical emergencies such as early and late infections. In particular, the presence of a caregiver ensuring surveillance in treatment compliance and interacting with the doctor is essential for a successful management of patients with the new agents.

With aging, many subjects exhibit a progressive decrease in glomerular filtration rate and renal blood flow [45]. In older patients with a decrease in the glomerular filtration rate, it is recommended that the fludarabine dose should be adjusted to the glomerular filtration rate in order to avoid an increased toxicity. In patients with a significant decrease in renal function, bendamustine should be considered as a better treatment option than fludarabine [46]. There is no information about the 
impact of age on the kinase inhibitors-related toxicity. It is expected that a liquid imbalance due to diarrhea could be clinically more relevant in older patients. Moreover, the use of anti-aggregants and anti-coagulants is more common in the aged population and this could increase the risk of bleeding events in patients treated with ibrutinib. AF, a common arrhythmia with increasing prevalence with aging, is expected to be more frequent in elderly patients treated with the BTK inhibitor ibrutinib [47].

\section{TREATMENT OPTIONS FOR ELDERLY AND UNFIT PATIENTS WITH CLL}

The efficacy and safety of regimens investigated in elderly and/or unfit patients will be reported and commented in this section. As for younger and fit patients, the absence/presence of the deletion and/or mutation of the TP53 gene should be checked also in elderly and/or unfit patients. These aberrations address the treatment choice independently of age and fitness status of CLL patients.

\subsection{Dose-reduced FCR regimens}

The FCR-Lite schedules include fludarabine and cyclophosphamide at a lower dose, given intravenously or orally and combined with rituximab given at the standard dose or with increased frequency [48-51]. Recently, Lew et al. [50] retrospectively reviewed the impact of dose reductions on the outcome of 42 consecutive patients with a median age of 72 years treated with the FCR regimen in the real life. Despite a median cumulative dose reduction of fludarabine by $50 \%$, the overall response (OR) and complete response $(C R)$ rates were $86 \%$ and $38 \%$, respectively (frontline OR and CR rates, $94 \%$ and $59 \%$; previously treated, $80 \%$ and $24 \%$ ). In addition, dose reductions of $25-75 \%$ were not significantly associated with an inferior PFS as compared to minimal reductions $(\leq 25 \%)$.

However, the reliability of data is limited by the retrospective nature of this study, the limited number of analyzed patients that did not permit the detection of statistically or clinically meaningful differences between the dosing groups. Different results have been reported by Kovaks et al.[51] in a pooled analysis of 635 patients who received frontline therapy with FCR within two prospective 
GCLLSG trials. This study indicated that a $>20 \%$ reduction of the planned dose of FCR, compromised significantly, not only PFS, but also OS.

A similar activity combined with a lower toxicity than the standard dose FCR have been reported with pentostatin and cyclophosphamide combined with rituximab [52].

\subsection{Bendamustine and rituximab (BR)}

In the randomized phase III CLL10 trial of the GCLLSG 564 fit patients without deletion 17p were randomized to receive the FCR or the BR regimen [4]. This study showed that the FCR regimen was significantly more effective than the $B R$ regimen in terms of $C R$ rate $(41 \%$ vs. $32 \%, p=0.026)$ and PFS (55.2 vs. 41.7 months, $p=0.001$ ). However, when older ( 65 years) patients were considered, there was no significant difference in PFS between patients treated with the FCR or the BR schedule (median not reached vs 48.5 months; $p=0.172$ ). In addition, severe granulocytopenia and infections were significantly more common with the FCR regimen than the BR regimen ( $88 \%$ vs. $68 \%, p<0.001$ and $40 \%$ vs. $25 \%, p=0.001)$. In particular, toxicity was more relevant in fit patients older than 65 years (grade $3-4$ infections, $48 \%$ vs. $27 \%, p=0.001$ ). These findings suggest that while the FCR regimen should be still considered the standard front-line treatment for fit patients younger than 65 years with no deletion and/or mutation of the TP53 gene, the BR regimen should be considered a better treatment option for fit patients older than 65 years. The results of Mable trial [53] also suggests that the BR regimen is an effective treatment option for older patients. In this study 241 patients were randomized to receive front-line treatment with rituximab combined with bendamustine $(B R)$ or chlorambucil (R-Clb). The median age was 72 years, and the median number of comorbidities three. CR rate was higher for the BR group (21\%) when compared with the R-Clb group $(7 \% ; \mathrm{p}<0.001)$. This translated in a significantly higher median PFS in patients treated with the BR regimen (39.6 months) than the R-Clb regimen (29.9 months; $p=0.003$ ). However, the rate of grade $\geq 3$ adverse events, in particular infections, was greater for the BR group when compared with the R-Clb group. 
The bendamustine and obinutuzumab combination is also an effective regimen that has been investigated in patients with a median age of 67.6 years with promising results, a CR rate of $32.3 \%$ with a MRD negative status in the bone marrow in $38.4 \%$ of cases [54].

\subsection{Chlorambucil-based treatments}

Before the introduction of fludarabine in the late $80^{\prime}$ chlorambucil was the only treatment for the majority of CLL patients. In a GCLLSG study patients $>65$ years of age (median, 70 years) were randomized to receive front-line treatment with fludarabine or chlorambucil [3]. Patients treated with fludarabine had better results in terms of OR and CR rates. However, no differences in the PFS and OS were observed between the two groups. Toxicity, in particular myelosuppression, was more marked in patients who received fludarabine. Similar results were reported also in a retrospective study by Wovach et al. [55] that analyzed the outcome of 663 patients included in first-line CALGB trials and treated with different regimens: chlorambucil, fludarabine, fludarabine plus rituximab (FR). PFS and OS were improved in the presence of a fludarabine-based regimen in patients younger than 70 years but not in older adults, while the addition of rituximab to fludarabine improved the outcome irrespective of age. These data suggested that in the setting of elderly patients there was no benefit from fludarabine compared to chlorambucil, while the addition of rituximab was beneficial regardless of age.

Chlorambucil, is associated with a low rate of complete responses and to a short response duration. In order to improve the outcome of patients not suitable for aggressive treatments, the activity of regimens combining chlorambucil with an anti-CD20 monoclonal antibody as front-line treatment in elderly and/or unfit patients has been investigated.

Hillmen et al. [17] carried out a phase II trial of chlorambucil combined with rituximab in 100 elderly patients with a median age of 70 years. The OR and CR rates were $84 \%$ and $10 \%$ with a median PFS of 23.5 months. Grade 3-4 granulocytopenia was observed in $41 \%$ of cases.

Foà et al. [18] evaluated the activity of the chlorambucil and rituximab combination as first-line induction treatment in patients over 65 years. Responders were then randomized to receive or not 12 doses of rituximab every 2 months or clinical observation. A response was recorded in $82.4 \%$ of 
patients, a CR in $16.5 \%$ and a CR with incomplete bone marrow recovery in $2.4 \%$. The median PFS was 34.7 months. Patients who received a maintenance treatment with rituximab showed a better PFS. Rituximab maintenance was most beneficial for patients in partial response and in unmutated IGHV cases. The presence of a TP53 abnormalities, complex karyotype and low CD20 gene expression predicted lack of response; the presence of a SF3B1 mutation and BIRC3 disruption were associated with low CR rates, while IGHV mutations significantly predicted PFS.

In a phase III GCLLSG study, 781 treatment naïve, unfit patients with a median age of 73 years were randomized to receive chlorambucil, rituximab plus chlorambucil, or obinutuzumab plus chlorambucil [19]. Patients treated with chlorambucil and obinutuzumab showed the best outcome in terms of OR, CR rate and PFS (ORR 77.3\%, CR 22.3\%, median PFS 26.7 months). In addition, a higher proportion of responding patients treated with chlorambucil and obinutuzumab achieved a MRD negative status in the peripheral blood and the bone marrow ( $38 \%$ and $20 \%$, respectively). In an updated analysys, treatment with chlorambucil combined with obinutuzumab was still associated with a better outcome in terms of PFS and time to the next treatment [56]. Grade 3/4 granulocytopenia was more frequently observed with chlorambucil plus obinutuzumab but was not associated with a higher infection rate. Grade 3/4 infusion-related reactions (IRRs) were more common with chlorambucil plus obinutuzumab. The IRRs were usually observed during the first administration. Premedication, dose splitting of the first dosage and prophylactically withholding of any anti-hypertensive medications were introduced to reduce the rate and severity of IRRs.

In a randomized study by Hillmen et al. [20] 447 patients not eligible for a fludarabine-based therapy and with a median age of 69 years were randomized to receive a front-line treatment with chlorambucil or with chlorambucil plus ofatumumab. Patients who received chlorambucil and ofatumumab showed a significantly better outcome than those treated with chlorambucil in terms of ORR (82\% vs $69 \%)$, CR rate (12\% vs $1 \%$ ) and PFS (median PFS, 22.4 vs 13.1 months).

\subsection{New agents}

During the last the last years, kinase inhibitors targeting the BCR signaling, ibrutinib, the Bruton tyrosine kinase (BTK) inhibitor, and idelalisib, the phosphatidylinositol-3-kinase (PI3K) inhibitor, 
have been widely investigated in patients with CLL. These agents have shown a high activity with a relatively good profile of toxicity in all age patients, in patients with adverse genetic characteristics and/or relapsed/refractory disease. The effectiveness of the BCR antagonists has led to their rapid license in the US and in several European countries for the treatment of patients with del(17p) and/or TP53 mutation and as salvage treatment of relapsed/refractory (R/R) patients. Recently, ibrutinib has been also approved as first-line treatment option for the front-line treatment of all patients with CLL. The Bcl2 inhibitor venetoclax (ABT-199) revealed also a very high activity in CLL and it has been recently approved in the US for the treatment of patients with CLL with $17 p$ deletion and who have been treated with at least one prior therapy. Novel agents targeting different kinases such as duvelisib and acalabrutinib showed promising results and are currently being tested in ongoing trials $[57,58]$. Because of their activity, the oral administration and the relatively favorable profile of toxicity, kinase and Bcl2 inhibitors represent a treatment option for older/unfit patients characterized by a genetic profile associated with a low response to chemoimmunotherapy or in whom chemoimmunotherapy is less well-tolerated.

\subsection{Ibrutinib}

Treatment with ibrutinib as a single agent, at a fixed dose of $420 \mathrm{mg} / \mathrm{day}$, showed a $90 \%$ ORR, $7 \%$ CR and a PFS at 30 months of $69 \%[59,60]$. In treatment-naïve (TN) CLL patients over 65 years the ORR was $84 \%$, with an estimated PFS at 30 months of $96 \%[22,60]$. Treatment was well tolerated and the most frequently AEs on ibrutinib were diarrhea, fatigue, infections and bleeding. Bleeding AEs were common, but usually mild, while severe adverse events (SAEs) resulting in treatment discontinuation were recorded in only a minority of cases. As expected, severe infections were more common in R/R than in TN patients. However, in patients on long-term treatment the rate of infections decreased over time $[22,59,60]$. In the randomized Resonate trial, R/R patients treated with ibrutinib showed a significantly better outcome that those who received ofatumumab [61]. Similarly, in the randomized Helios study patients treated with ibrutinib plus BR showed a better outcome with regard to response rates and PFS than those treated with BR [47]. The randomized Resonate-2 phase 3 trial was carried out in 269 TN patients aged $\geq 65$ years, not 
eligible for an aggressive chemoimmunotherapy [23]. Patients with deletion 17p and those receiving warfarin were excluded from the study. The median age of patients was 73 years and $70 \%$ of them were aged $>70$ years. The ORR rate was significantly higher for patients treated with ibrutinib than chlorambucil $(86 \%$ vs $35 \% ; p<0.001)$. The median PFS was not reached in the ibrutinib group while it was 18.9 months in the chlorambucil group $(P<.001)$. In addition, ibrutinib significantly prolonged OS, that was at 24 months, $98 \%$ for patients treated with ibrutinib versus $85 \%$ for those treated with chlorambucil $(p=.001)$. In addition, ibrutinib significantly prolonged os, that was at 24 months, $98 \%$ for patients treated with ibrutinib versus $85 \%$ for those treated with chlorambucil $(p=.001)$. These results have lead to the recent the approval of ibrutinib in the US and in Europe as single agent in the front-line treatment of patients with CLL. In the Resonate-2 study, toxicity was usually mild and the most common AEs on ibrutinib were diarrhea, fatigue, cough and nausea [23].

Across different studies, AF, was found to occur more frequently in patients treated with ibrutinib ( $4 \%$ ibrutinib vs. $1 \%$ ofatumumab; ibrutinib plus bendamustine and rituximab, $7 \%$ vs. bendamustine and rituximab, $2.8 \%$; ibrutinib, $6 \%$ vs. chlorambucil, $1 \%$ ) $[61,47,23]$. However, only a minority of patients with AF required ibrutinib discontinuation.

Hypertension, another common problem of aged population, has been recorded in patients on long-term treatment with ibrutinib, with a rate in TN and R/R patients of $7 \%$ and $20 \%$, respectively [60]. Since in older patients AF, hypertension, concomitant anti-coagulation treatment may be a concern when making a treatment decision, a close collaboration between cardiologists and hematologists is recommended in order to identify not only specific risk factors for AEs but also their appropriate management. Randomised controlled studies should be carried out in elderly patients to compare the activity and safety of ibrutinib with that of regimens including chlorambucil combined with an anti-CD20 monoclonal antibody, obinutuzumab, rituximab, obinutuzumab

\subsection{Acalabrutinib}

A more selective BTK inhibitor, acalabrutinib, was given in a phase 1-2 multicenter study to 61 relapsed patients with CLL with a relatively young median age, 62 years. A high ORR was 
observed, $95 \%$ (85\% partial responses and 10\% partial response with lymphocytosis). Among patients with deletion $17 p$ the ORR was $100 \%$. The mild toxicity associated with this more specific BTK inhibitor is an appealing aspect. In particular, no severe bleeding events or AF were recorded with acalabrutinib [58]. This is not a negligible aspect to consider for the treatment of elderly patients who are frequently on anti-coagulant or anti-aggregant agents and have an increased risk of $A F$.

\subsection{Idelalisib}

The activity of idelalisib - the selective, oral inhibitor blocking the delta isoform of the enzyme phosphoinositide 3-kinase (PI3Kס) - was investigated in the multicenter, randomized, double-blind, placebo-controlled, phase 3 study by Furman et al. in 220 R/R patients with CLL [24]. Patients received rituximab and idelalisib or rituximab and placebo twice daily. The median age of patients was 71 years and the median number of prior treatments, three. In addition, $40 \%$ of patients had at least a moderate renal dysfunction (creatinine clearance, $<60 \mathrm{ml}$ per minute), $35 \%$ a poor bone marrow function (grade 3 or higher anemia, thrombocytopenia or neutropenia) and $85 \%$ a CIRS score higher than six, Deletion 17p or TP53 mutations were present in $40 \%$ of patients. The study was stopped early on the recommendation of the data and safety monitoring board owing to overwhelming efficacy. Patients receiving idelalisib showed not only a significantly higher ORR than those treated with rituximab and placebo ( $81 \%$ vs. $13 \% ; P<0.001)$, but also a significantly better OS (at 12 months, $92 \%$ vs. $80 \%$; $\mathrm{P}=0.02$ ) with a median PFS not reached in the idelalisib group and 5.5 months in the placebo plus rituximab group $(\mathrm{P}<0.001)$. The most frequent $(>30 \%)$ AEs (any grade) in patients treated with idelalisib were diarrhea and colitis (64\%), rash (58\%), pyrexia (42\%), nausea (38\%), chills (36\%), cough (33\%) and fatigue $(31 \%)$. Grade $\geq 3$ transaminitis was recorded in $23 \%$ of patients.

O'Brien et al. [25] reported the first clinical trial of idelalisib with rituximab as initial treatment of 64 CLL/SLL patients with a median age of 71 years. Idelalisib plus rituximab was highly effective with an ORR of $95 \%$, a CR rate of $19 \%$ and a PFS at 36 months of $83 \%$. The most frequent treatmentlimiting $\mathrm{AE}$ of any grade was diarrhea, while elevated transaminases were recorded in $67 \%$ of patients $(23 \%$ grade $\geq 3)$. Thomson et al. [62] analyzed the long-term follow-up data from $40 \mathrm{TN}$ 
patients aged $\geq 65$ years who received idelalisib combined with rituximab at the MD Anderson Cancer Center. The study focused on the outcome of patients who permanently ceased idelalisib for toxicity (diarrhea/colitis, 7 patients; treatment-related pneumonitis, 3). This small series of patients had a higher risk of disease progression.

The idelalisib-related pattern of toxicity - namely colitis, hepatitis and pneumonitis $[63,64]-$ is consistent across studies, usually regresses on steroids and is more frequently observed in younger and previously untreated patients. Biopsies of idelalisib-induced colitis were typically characterized by CD8+ T-cell infiltrates associated with crypt apoptosis [65, 66]. Furthermore, in patients who experienced transaminitis a decrease in the T-regulatory cell count of has been considered as a direct effect of idelalisib on the survival and function of regulatory $T$ cells [67]. Genetic mutations that disrupt regulatory T-cell function are associated in mice and humans with a similar autoimmune syndrome of hepatitis, enteritis and pneumonitis [68].

Recently, six clinical trials carried out in patients with CLL, small lymphocytic lymphoma and indolent non-Hodgkin lymphomas and exploring idelalisib in combination with other drugs, have been halted by the FDA due to an increased rate of AEs and death, mostly due to infections.

After a safety review, the European Medicines Agency's (EMA's) Pharmacovigilance Risk Assessment Committee (PRAC) confirmed that the benefits of treatment with idelalisib outweight the risks [69] However, the PRAC did confirm that treatment with idelalisib carries an increased risk for serious infections and that treatment should not be started in patients with a generalized infection. The PRAC recommended that all patients treated with idelalisib should be given antibiotics to prevent Pneumocystis jirovecii pneumonia during treatment and for up to 2 to 6 months after stopping treatment. The committee recommended also that patients on idelalisib should be monitored for infections, CMV reactivation and have regular blood tests for white cell counts.

In the US and Europe, the use of idelalisib in combination with rituximab is authorized for the treatment of CLL patients who have received previous treatment. In Europe, idelalisib in combination with rituximab is authorized for the treatment of CLL patients who have received previous treatment and in previously untreated patients with deletion 17p or TP53 mutation 
provided they cannot take any alternative treatment and that the measures agreed to prevent infection are followed. At present, regimens including idelalisib in combination with other agents should not be used in patients with CLL.

\subsection{Venetoclax}

Venetoclax is an oral selective inhibitor of the pro-survival protein $\mathrm{Bcl}-2$ that directly induces apoptosis independently of TP53 and therefore restores the apoptotic ability of leukemic cells. This agent clears the blood and bone marrow more effectively than the kinase inhibitors and induced a CR with no residual disease in a relevant proportion of $R / R$ patients even in those with deletion $17 \mathrm{p}$.

In the phase I and expansion cohort study, 116 heavily pre-treated patients with CLL and a median age of 66 years treated with venetoclax showed OR and CR rates of $79 \%$ and $20 \%$, respectively, with a median PFS of 25 months in the dose-escalation cohorts [70].

In a study including 49 R/R patients with a median age of 68 years, venetoclax was combined with rituximab [71]. A high rate of responses, $86 \%$, and CRs, $47 \%$, with a $24-$ month PFS of $83 \%$, were reported. Similar response rates were observed also in patients with deletion 17p. In addition, more than half responding patients showed no residual disease in the bone marrow.

Previously untreated patients with coexisting medical conditions and a median age of 75 years were included in the safety run-in phase of the CLL14 trial of the GCLLSG group to assess the tolerability of obinutuzumab combined with venetoclax [72]. Preliminary results of this study in terms of safety are very encouraging. A strong limitation of this agent was initially represented by the tumor lysis syndrome (TLS). Because of the decrease in renal function in the elderly, the use of venetoclax is a cause of concern. However, the introduction of a gradual venetoclax ramp schedule $(20,50,100,200,400 \mathrm{mg})$ over 4-5 weeks, TLS prophylaxis, and close monitoring of patients prevented and mitigated tumor lysis syndrome [73].

\subsection{Patients with deletion 17p/TP53 mutations}

The presence of deletion 17p and/orTP53 mutations are associated with poor response to chemoimmunotherapy and short survival. During the last decade, the anti-CD52 antibody 
alemtuzumab was considered as the best treatment option for this subset of patients. Alemtuzumab as a single agent or combined with steroids was associated with relatively high response rates $[74,75]$. However, patients treated with alemtuzumab showed also a high morbidity and mortality due to severe infections. The only treatment option resulting in a better outcome was an allogeneic transplant that cannot be considered in older/unfit patients.

The introduction of the new agents has changed significantly the outcome of these patients who show with the BCR and BCL-2 inhibitors a better outcome compared to what observed with all previously used regimens. At present, there is a general agreement that, independently of age, these agents represent the treatment of choice for all CLL patients with deletion 17p and/or TP53 mutations $[24,59,60,70,71,76-78]$.

Two studies have recently focused on patients with deletion $17 \mathrm{p}$. Venetoclax was investigated in $107 \mathrm{R} / \mathrm{R}$ patients with CLL and deletion $17 \mathrm{p}$ in a phase 2, single-arm, multicenter study [78]. Patients received venetoclax with a weekly dose ramp-up schedule over 4-5 weeks. Patients were then given daily $400 \mathrm{mg}$ continuous dosing until disease progression or discontinuation for another reason. A response was achieved by $79.4 \%$ of patients with a PFS and OS at 12 months of $72 \%$ and $87 \%$, respectively. Based on these results, venetoclax has been approved as monotherapy for patients with CLL with a $17 p$ deletion who have received at least one prior therapy, and is awaiting approval for similar indications in the EU and Canada.

The second study focused on patients with deletion $17 p$, is the Resonate-17 study. This study enrolled 147 R/R patients who were treated with ibrutinib as single agent. Similarly, high response rates were obtained, with an ORR of $82.6 \%$ and a PFS at 12 months of $79.3 \%$ [76].

\subsection{Very old patients with CLL}

The number of really old ( $\geq 80-85$ years) CLL patients with a life expectancy of just a few years, is increasing. Very old patients are frequently characterized by a high number of comorbidities and disabilities and are not eligible for toxic treatments or agents requiring close monitoring. No clinical trials have specifically focused on this subset of patients. A conservative therapy with manageable drugs, such as oral alkylating agents, could be a reasonable treatment approach in the majority of 
these cases. The cooperation between hematologists, family physicians and caregivers is crucial in order to realize the optimal treatment management of these patients.

\section{THE NCCN AND ESMO GUIDELINES FOR THE FRONT-LINE TREATMENT OF ELDERLY/UNFIT CLL PATIENTS}

The last NCCN and ESMO guidelines [32,79] recommend in patients with CLL different front-line treatment approaches according to the presence of deletion $17 \mathrm{p} /$ TP53 mutations, the fitness status and age of patients (Table 3 ). In both guidelines, a regimen combining chlorambucil with an antiCD20 monoclonal antibody (obinutuzumab in the 2016 NCCN guidelines) is considered the first treatment option for the front-line management of patients without deletion $17 \mathrm{p}$ and/or TP53 mutations. Based on the data from the randomized Resonate- 2 trial and the FDA approval, the 2016 NCCN guidelines consider ibrutinib as a front-line treatment option for the treatment of all patients with CLL. Ibrutinib in the 2016 NCCN guidelines and a BCR inhibitor in the 2015 ESMO guidelines, are recommended as first line treatment option for patients with deletion $17 p$ and/or TP53 mutations.

\section{EXPERT COMMENTARY}

During the last years, $\mathrm{BCR}$ antagonists and the $\mathrm{Bcl}-2$ inhibitor venetoclax have changed the therapeutic landscape of CLL with a marked improvement of outcome. It appears likely that the chemo-free therapy will revolutionize the management of CLL and will soon be considered as the preferable option for the front-line treatment of a large proportion of patients with CLL including elderly and unfit patients.

\section{FIVE-YEAR'S VIEW}

Many aspects of the treatment of CLL patients will need to be evaluated during the next few years. The best treatment options for patients with no 17p deletions and/or TP53 mutations should be still defined, according to the clinical and biologic profile of each case in order to select patients likely 
to achieve a long PFS after chemoimmunotherapy and those who may have a better outcome with a chemo-free approach.

An observational, multicenter, retrospective study by Rossi et al. [80], included 404 patients with progressive disease who received FCR frontline outside clinical trials. According to the combination of three biomarkers tested before treatment, IGHV mutation status, 11q deletion and $17 p$ deletion, a very low-risk category of patients was identified. Patients carrying mutated IGHV genes, but neither $11 q$ or $17 p$ deletion, showed a life expectancy of $91 \%$ at 5 years, superimposable to that observed in the matched normal general population.

The excellent outcome of mutated patients was also observed in two clinical trials that included selected patients treated with FCR. In the CLL8 study more than $83 \%$ of patients with mutated IGHV treated with FCR were still alive after almost 6 years of observation time [81]. This excellent outcome was showed by all cytogenetic subgroups, except for patients with deletion $17 p$ and normal karyotype. Similarly, at the MD Anderson Cancer Center, IGVH mutated patients showed a PFS of $53.9 \%$ at 12.8 years [82]. About half of IGVH mutated patients achieved MRD negativity post-treatment with a PFS of $79.8 \%$ at 12.8 years. In addition, a plateau was seen on the PFS curve in patients with mutated IGHV, with no relapses beyond 10.4 years in 42 patients.

The significant prognostic effect of the IGVH mutational status emerged also in the CLL11 trial [19] including unfit/elderly patients treated with chlorambucil-based chemoimmunotherapy regimens that are better tolerated than the FCR regimen in this subset of patients. In multivariate analysis unmutated IGHY, deletion 17p and/or TP53 mutations were identified as independent factors for PFS while complex karyotypes and POT1 mutations (8.1\% of patients) represented significant prognostic factors for an unfavorable survival, independently of the IGHV mutation status [83].

These observations suggest that in CLL, independently of age, the therapeutic approach should be guided by the genetic profile. Prospective clinical trials, comparing FCR to the new targeted agents, should be designed in order to identify the optimal treatment approach for CLL patients with a favorable genetic profile. 
Probably, a treatment with new agents could be a better strategy for other subsets of patients with an adverse genetic profile, and a lower susceptibility to chemotherapy. Factors predicting the early and long-term toxicity of new agents and their appropriate management should be also identified. The optimal combinations, the appropriate sequencing of the new agents, the duration of treatment, mechanisms leading to resistance, need to be explored. In addition, the activity and safety of a multitude of novel agents is on investigation in ongoing trials.

The cost of cancer treatment is dramatically increasing and has become a matter of international attention. Treatment costs are also a relevant aspect in the management of patients with CLL, the most diffuse form of leukemia in the adult population of the western world. Until now, guidelines have not considered costs and the relative cost effectiveness of treatment options in the development of recommendations. In the near future, clinical practice guidelines, in addition to clinical and biologic criteria, will also need to include costs and cost-effectiveness information for each recommended treatment option. Recommendations considering both the clinical ad economic impact of the different treatment approaches will enable informed decisions ensuring the optimal treatment choice in the context of the real-world constraints and the health care policies developed in each country.

\section{CONCLUSIONS}

Over the years, the management of CLL has changed profoundly following the advent of treatment regimens that have markedly impacted on the clinical outcome, including that of elderly patients with reduced organ function. The activity and toxicity of different chemoimmunotherapy schedules has been specifically investigated in patients with comorbidities. More recently, agents with targeted mechanisms of action and less toxicity than chemoimmunotherapy have clearly shown to be very active also in elderly/unfit patients.

At present, multiple treatment options are available in many countries allowing treating physiciancs to select the optimal treatment for each patient. Age, comorbidities and the biological profile of the leukemic cells should be taken into consideration during the decision-making process to address the treatment choice of CLL patients. 


\section{KEY ISSUES}

- About $75 \%$ of patients with chronic lymphocytic leukemia (CLL) are more than 65 years at the time of diagnosis.

- CLL, and no other age-related diseases, is the most frequent cause of death for the majority of older patients.

- The progressive reduction in the functional reserve of multiple organ systems affects both pharmacokinetics and pharmacodynamics of chemotherapy leading to an increased risk of treatment complications.

- An appropriate assessment of comorbidities should be considered in the pre-treatment work-up of elderly/unfit patients to estimate the physiological age and the more appropriate treatment approach.

- The best treatment option should consider the clinical and biologic profile of each case in order to select patients likely to achieve a long PFS after chemoimmunotherapy and those who may have a better outcome with a chemo-free approach.

- As for younger and fit patients, in elderly and/or unfit patients the absence/presence of deletions and/or mutations of the TP53 gene should be investigated at the time of treatment (and re-treatment).

- At present, regimens including chlorambucil combined with an anti-CD20 monoclonal antibody and the BTK inhibitor, ibrutinib, effective also in patients with deletion 17p/TP53 mutations are considered optimal treatment options for elderly/unfit patients with CLL.

\section{Funding}

This paper was not funded.

\section{Declaration of Interest}


F Mauro and R Foà are members of advisory boards for Roche, Janssen, Gilead and Abbvie. The authors have no other relevant affiliations or financial involvement with any organization or entity with a financial interest in or financial conflict with the subject matter or materials discussed in the manuscript apart from those disclosed.

\section{REFERENCES}

* Article of importance

** Article of considerable importance

1. Satram-Hoang $S$, Reyes $C$, Hoang $K Q$ et al. Treatment practice in the elderly patient with chronic lymphocytic leukemia-analysis of the combined SEER and Medicare database. Ann Hematol. 2014; 93(8):1335-44.

2. Baumann T, Delgado J, Santacruz R et al. Chronic lymphocytic leukemia in the elderly: clinico-bilogical features, outcomes, and proposal of a prognostic model. Haematologica. 2014;99(10):1599-604.

3. Eichhorst BF, Busch R, Stilgenbauer S et al. First-line therapy with fludarabine compared with chlorambucil does not result in a major benefit for elderly patients with advanced chronic lymphocytic leukemia. Blood 2009; 114(16):3382-91. ${ }^{* *}=$ of considerable importance. Front-line treatment with fludarabine compared to chlorambucil was not associated with a major clinical benefit in CLL patients older than 65 years.

4. Eichhorst B, Fink AM, Bahlo J et al. First-line chemoimmunotherapy with bendamustine and rituximab versus fludarabine, cyclophosphamide, and rituximab in patients with advanced chronic lymphocytic leukaemia (CLL10): an international, open-label, randomised, phase 3, non-inferiority trial. Lancet Oncol 2016 [Epub ahead of print] doi: 10.1016/S14702045(16)30051-1

5. Badoux XC, Keating MJ, Wen S et al. Lenalidomide as initial therapy of elderly patients with chronic lymphocytic leukemia. Blood 2011;118 (13):3489-98.

6. Balducci L, Dolan D. Chronic Lymphocytic Leukemia in the Elderly: Epidemiology and Proposed Patient-Related Approach. Cancer control 2015; 22(4 Suppl):3-6.

7. Tam CS, O'Brien S, Wierda W et al. Long-term results of the fludarabine, cyclophosphamide, and rituximab regimen as initial therapy of chronic lymphocytic leukemia. Blood 2008; 112(4):975-80. 
8. Fischer $\mathrm{K}$, Cramer $\mathrm{P}$, Busch $\mathrm{R}$ et al. Bendamustine in combination with rituximab for previously untreated patients with chronic lymphocytic leukemia: a multicenter phase II trial of the German Chronic Lymphocytic Leukemia Study Group. J Clin Oncol 2012;30(26):3209-16.

9. Goede V, Cramer P, Busch R et al. Interactions between comorbidity and treatment of chronic lymphocytic leukemia: results of German Chronic Lymphocytic Leukemia Study Group trials. Haematologica 2014; 99(6):1095-100 . ${ }^{* *}=$ of considerable importance. This study investigated the impact of comorbidity in 555 CLL patients enrolled in two German trials and identified comorbidity as an independent predictor of adverse outcome.

10. Abrisqueta $P$, Pereira $A$, Rozman $C$ et al. Improving survival in patients with chronic lymphocytic leukemia (1980-2008): the Hospital Clinic of Barcelona experience. Blood 2009;114(10):2044-50.

11. Brenner $\mathrm{H}$, Gondos $A$, Pulte D. Trends in long-term survival of patients with chronic lymphocytic leukemia from the 1980 s to the early 21 st century. Blood 2008;111(10):491621.

12. Wierda WG, O'Brien $S$, Wang $X$ et al. Characteristics associated with important clinical end points in patients with chronic lymphocytic leukemia at initial treatment. J Clin Oncol 2009;27(10):1637-43.

13. Shanafelt TD, Rabe KG, Kay NE et al. Age at diagnosis and the utility of prognostic testing in patients with chronic lymphocytic leukemia. Cancer 2010; 116 (20):4777-87.

14. Mauro FR, Foa R, Giannarelli D et al. Clinical characteristics and outcome of young chronic lymphocytic leukemia patients: a single institution study of 204 cases. Blood 1999;94(2):448-54.

15. Gentile M, Mauro FR, Rossi D et al. Italian external and multicentric validation of the MD Anderson Cancer Center nomogram and prognostic index for chronic lymphocytic leukaemia patients: analysis of 1502 cases. Br J Haematol 2014;167(2):224-32.

16. International CLL-IPI working group. An international prognostic index for patients with chronic lymphocytic leukaemia (CLL-IPI): a meta-analysis of individual patient data. Lancet Oncol 2016;17(6):779-90.

17. Hillmen P, Gribben JG, Follows GA et al. Rituximab plus chlorambucil as first-line treatment for chronic lymphocytic leukemia: Final analysis of an open-label phase II study. J Clin Oncol 2014; 32(12):1236-41. 
18. Foa R, Del Giudice I, Cuneo A et al. Chlorambucil plus rituximab with or without maintenance rituximab as first-line treatment for elderly chronic lymphocytic leukemia patients. Am J Hematol 2014;89(5):480-86.

19. Goede V, Fischer K, Busch $R$ et al. Obinutuzumab plus chlorambucil in patients with CLL and coexisting conditions. N Engl J Med 2014; 370(12):1101-10. ${ }^{* *}=$ of considerable importance. Combining an anti-CD20 antibody with chlorambucil improved outcomes in patients with CLL and coexisting comorbidities. Obinutuzumab was superior to rituximab when each was combined with chlorambucil.

20. Hillmen $P$, Robak T, Janssens $A$ et al. Chlorambucil plus ofatumumab versus chlorambucil alone in previously untreated patients with chronic lymphocytic leukaemia (COMPLEMENT 1): a randomised, multicentre, open-label phase 3 trial. Lancet 2015; 385(9980):1873-83. ${ }^{* *}=$ of considerable importance. In CLL who were elderly or had comorbidities, ofatumumab combined with chlorambucil was a more effective front-line regimen than chlorambucil with a manageable side-effect profile.considerable importance.

21. Strati $P$, Keating MJ, Wierda WG et al. Lenalidomide induces long-lasting responses in elderly patients with chronic lymphocytic leukemia. Blood 2013;122(5):734-37.

22. O'Brien S, Furman RR, Coutre SE et al. Ibrutinib as initial therapy for elderly patients with chronic lymphocytic leukaemia or small lymphocytic lymphoma: an open-label, multicentre, phase 1b/2 trial. Lancet Oncol 2014;15 (1):48-58. * of importance. This study that describes safety and activity of ibrutinib in elderly, previously untreated patients with CLL or small lymphocytic lymphoma.

23. Burger JA, Tedeschi A, Barr PM et al. Ibrutinib as Initial Therapy for Patients with Chronic Lymphocytic Leukemia. N Engl J Med 2015; 373 (25):2425-37. **= of considerable importance. In patients $\geq 65$ years with CLL or small lymphocytic lymphoma, ibrutinib was superior to chlorambucil.

24. Furman RR, Sharman JP, Coutre SE et al. Idelalisib and rituximab in relapsed chronic lymphocytic leukemia. N Engl J Med 2014; 370(11):997-1007. ${ }^{* *}=$ of considerable importance. The combination of idelalisib and rituximab, as compared with placebo and rituximab, significantly improved the outcome of patients with relapsed CLL who were less able to undergo chemotherapy.

25. O'Brien SM, Lamanna N, Kipps TJ et al. A phase 2 study of idelalisib plus rituximab in treatment-naive older patients with chronic lymphocytic leukemia. Blood 2015; 126(25):2686-94. * = of importance. This prospective, study describes in CLL patients, $\geq 65$ years, the activity and safety of the idelalisib and rituximab regimen given as front-line therapy.

26. Jagger A, Shimojima Y, Goronzy JJ, Weyand CM. Regulatory T cells and the immune aging process: a mini-review. Gerontology 2014; 60(2):130-7.

27. Balducci L. Myelosuppression and its consequences in elderly patients with cancer. Oncology 2003; 17(Suppl 11):27-32.

28. Read WL, Tierney RM, Page NC et al. Differential prognostic impact of comorbidity. J Clin Oncol 2004;2(15):3099-103. 
29. Thurmes $P$, Call $T$, Slager $S$ et al. Comorbid conditions and survival in unselected, newly diagnosed patients with chronic lymphocytic leukemia. Leukemia Lymphoma 2008;49(1):49-56.

30. Charlson M, Szatrowski TP, Peterson J, Gold J. Validation of a combined comorbidity index. J Clin Epidemiol 1994;47(11):1245-51.

31. Miller MD, Paradis CF, Houck PR et al. Rating chronic medical illness burden in geropsychiatric practice and research: application of the Cumulative Illness Rating Scale. Psychiat Res 1992; 41(3): 237-48.

32. NCCN Cancer Network. Clinical Practice Guidelines in Oncology v3.2016 (NCCN Guidelines $\left.{ }^{\circledR}\right)$ - Chronic Lymphocytic Leukemia/Small Lymphocytic lymphoma, https://www.nccn.org/

33. Wierda WG, O'Brien S, Wang $X$ et al. Prognostic nomogram and index for overall survival in previously untreated patients with chronic lymphocytic leukemia. Blood 2007;109(11):4679-85.

34. Pflug N, Bahlo J, Shanafelt TD, et al. Development of a comprehensive prognostic index for patients with chronic lymphocytic leukemia. Blood 2014;124 (1):49-62.

35. Kutsch N, Bahlo J,Byrd JC,e tal. The International Prognostic Index for patients with CLL (CLL-IPI):ani nternationa Imeta-analysis.J Clin Oncol 2015; 33 (suppl; abstr 7002).

36. Parikh SA, Shanafelt TD. Prognostic factors and risk stratification in chronic lymphocytic leukemia. Semin Oncol. 2016;43(2):233-40.

37. Naeim A, Aapro M, Subbarao R, Balducci L. Supportive care considerations for older adults with cancer. J Clin Oncol 2014;32(24):2627-34.

38. Wang L, Baser O, Kutikova $L$ et al. The impact of primary prophylaxis with granulocyte colony-stimulating factors on febrile neutropenia during chemotherapy: a systematic review and meta-analysis of randomized controlled trials. Support Care Cancer 2015;23(11):313140.

39. Aapro MS, Bohlius J, Cameron DA et al. 2010 update of EORTC guidelines for the use of granulocyte-colony stimulating factor to reduce the incidence of chemotherapy-induced febrile neutropenia in adult patients with lymphoproliferative disorders and solid tumours. Eur J Cancer 2011: 47(1):8-32.

40. Kuderer NM, Dale DC, Crawford J et al. Mortality, morbidity, and cost associated with febrile neutropenia in adult cancer patients. Cancer 2006;106(10):2258-66.

41. Morrison VA. Infectious complications of chronic lymphocytic leukaemia: pathogenesis, spectrum of infection, preventive approaches. Best Pract Res CI Ha 2010;23(1):145-53.

42. Balducci L. Anemia, fatigue and aging. Transfus Clin Biol 2010;17(5-6):375-81.

43. Shanafelt TD, Drake MT, Maurer MJ et al.Vitamin D insufficiency and prognosis in chronic lymphocytic leukemia. Blood 2011;117(5):1492-98.

44. Molica S1, Digiesi G, Antenucci A et al. Vitamin D insufficiency predicts time to first treatment (TFT) in early chronic lymphocytic leukemia (CLL). Leuk Res. 2012;36(4):443-7. 
45. Weinstein JR, Anderson S. The aging kidney: physiological changes. Adv Chronic Kidney Dis 2010;17(4):302-07.

46. Cheson BD, Brugger W, Damaj G et al. Optimal use of bendamustine in hematologic disorders: Treatment recommendations from an international consensus panel-an update. Leukemia Lymphoma 2016;57(4):766-82.

47. Chanan-Khan A, Cramer $P$, Demirkan $F$ et al. Ibrutinib combined with bendamustine and rituximab compared with placebo, bendamustine, and rituximab for previously treated chronic lymphocytic leukaemia or small lymphocytic lymphoma (HELIOS): a randomised, double-blind, phase 3 study. Lancet Oncol 2015; 17(2): 200-11.

48. Foon KA, Mehta D, Lentzsch S et al. Long-term results of chemoimmunotherapy with lowdose fludarabine, cyclophosphamide and high-dose rituximab as initial treatment for patients with chronic lymphocytic leukemia. Blood 2012; 119(13):3184-85.

49. Dartigeas C, Van Den Neste E, Berthou C et al. Evaluating abbreviated induction with fludarabine, cyclophosphamide, and dose-dense rituximab in elderly patients with chronic lymphocytic leukemia. Leukemia Lymphoma 2015; Sep 28:1-7 [Epub ahead of print]

50. Lew TE, Cheah CY, Carney DA et al. Dose-reduced fludarabine, cyclophosphamide and rituximab is well tolerated in older patients with chronic lymphocytic leukemia and has preserved therapeutic efficacy. Leukemia Lymphoma 2016;57(5):1044-53.

51. Kovacs G, Bahlo J, Kluth S et al. Prognostic Impact and Risk Factors of Reducing Prescribed Doses of Fludarabine, Cyclophosphamide and Rituximab (FCR) during Frontline Treatment of Chronic Lymphocytic Leukemia (CLL). Blood 2015 126:4156.

52. Shanafelt TD, Lin T, Geyer SM et al. Pentostatin, cyclophosphamide, and rituximab regimen in older patients with chronic lymphocytic leukemia. Cancer 2007; 109(11):229198.

53. Michallet AS, Aktan M, Schuh A, et al. Rituximab in combination with bendamustine or chlorambucil for the treatment of chronic lymphocytic leukemia: Primary results from the randomized phase IIlb MaBLe study. iwCLL Abstracts 2015:178.

54. Stilgenbauer S, MD PhD1, Ilhan O, Woszczyk D et al. Safety and Efficacy of Obinutuzumab Plus Bendamustine in Previously Untreated Patients with Chronic Lymphocytic Leukemia: Subgroup Analysis of the Green Study. Blood 2015; Blood 2015 126:493.

55. Woyach JA, Ruppert AS, Rai K et al. Impact of age on outcomes after initial therapy with chemotherapy and different chemoimmunotherapy regimens in patients with chronic Iymphocytic leukemia: results of sequential cancer and leukemia group B studies. J Clin Oncol 2013;31(4):440-47.

56. Goede V, Fischer K, Busch R et al. Chemoimmunotherapy with GA101 plus chlorambucil in patients with chronic lymphocytic leukemia and comorbidity: results of the CLL11 (BO21004) safety run-in. Leukemia 2013; 27(5):1172-74.

57. Balakrishnan K, Peluso M, Fu M et al. The phosphoinositide-3-kinase (PI3K)-delta and gamma inhibitor, IPI-145 (Duvelisib), overcomes signals from the PI3K/AKT/S6 pathway and promotes apoptosis in CLL. Leukemia 2015;29(9):1811-22.

58. Byrd JC, Harrington B, O'Brien S et al. Acalabrutinib (ACP-196) in Relapsed Chronic Lymphocytic Leukemia. New Engl J Med 2016; 374 (4):323-32. 
59. Byrd JC, Furman RR, Coutre SE et al. Targeting BTK with ibrutinib in relapsed chronic lymphocytic leukemia. New Engl J Med 2013;369(1):32-42.

60. Byrd JC, Furman RR, Coutre SE et al. Three-year follow-up of treatment-naive and previously treated patients with CLL and SLL receiving single-agent ibrutinib. Blood 2015;125(16):2497-506.

61. Byrd JC, Brown JR, O'Brien S et al. Ibrutinib versus ofatumumab in previously treated chronic lymphoid leukemia. N Engl J Med 2014; 371 (3):213-23.

62. Thompson PA, Stingo F, Keating MJ et al. Outcomes of patients with chronic lymphocytic leukemia treated with first-line idelalisib plus rituximab after cessation of treatment for toxicity. Cancer. 2016 May 16 [Epub ahead of print] doi: 10.1002/cncr.30069

63. Lampson BL, Kasar SN, Matos TR et al. Idelalisib given front-line for treatment of chronic lymphocytic leukemia causes frequent immune-mediated hepatotoxicity. Blood 2016; May 31. pii: blood-2016-03-707133 [Epub ahead of print]

64. Coutre SE, Barrientos JC, Brown JR et al. Management of adverse events associated with idelalisib treatment: expert panel opinion. Leukemia Lymphoma 2015;56(10):2779-86.

65. Louie CY, DiMaio MA, Matsukuma KE et al. Idelalisib-associated Enterocolitis:Clinicopathologic Features and Distinction From Other Enterocolitides. Am J Surg Pathol 2015; 39(12):1653-60.

66. Weidner AS, Panarelli NC, Geyer JT et al. Idelalisib-associated Colitis: Histologic Findings in 14 Patients. Am J Surg Pathol 2015;39(12):1661-67.

67. Patton DT, Garden OA, Pearce WP et al. Cutting edge: the phosphoinositide 3-kinase p110 delta is critical for the function of CD4+CD25+Foxp3+ regulatory T cells. J Immunol 2006;177(10):6598-602.

68. Ramsdell F, Ziegler SF. FOXP3 and scurfy: how it all began. Nat Rev Immunol. 2014;14(5):343-9.

69. http://www.ema.europa.eu/docs/en_GB/document_library/Press_release/2016/07/WC5002 09936.pdf

70. Roberts AW, Davids MS, Pagel JM et al. Targeting BCL2 with Venetoclax in Relapsed Chronic Lymphocytic Leukemia. N Engl J Med 2016;374(4):311-22.

71. Ma S BD, Seymour JF, et al. Deep and durable responses following venetoclax (ABT199/GDC-0199) combined with rituximab in patients with relapsed/refractory chronic lymphocytic leukemia: results from a phase 1b study. Blood 2015;126: 830.

72. Fischer K, Bishop H, et al. Results of the Safety Run-in Phase of CLL14 (BO25323): A Prospective, Open-Label, Multicenter Randomized Phase III Trial to Compare the Efficacy and Safety of Obinutuzumab and Venetoclax (GDC-0199/ABT-199) with Obinutuzumab and Chlorambucil in Patients with Previously Untreated CLL and Coexisting Medical Conditions. Blood 2015;126: 496. 
73. Seymour JF. Effective mitigation of tumor lysis syndrome with gradual venetoclax dose ramp, prophylaxis, and monitoring in patients with chronic lymphocytic leukemia. Ann Hematol. 2016 ;95(8):1361-2.

74. Stilgenbauer S, Zenz T, Winkler D et al. Subcutaneous alemtuzumab in fludarabinerefractory chronic lymphocytic leukemia: clinical results and prognostic marker analyses from the CLL2H study of the German Chronic Lymphocytic Leukemia Study Group. J Clin Oncol 2009;27(24):3994-4001.

75. Pettitt AR, Jackson R, Carruthers $S$ et al. Alemtuzumab in combination with methylprednisolone is a highly effective induction regimen for patients with chronic lymphocytic leukemia and deletion of TP53: fina I results of the national cancer research institute CLL206 trial. J Clin Oncol 2012;30(14):1647-55.

76. Stilgenbauer S, Ilhan O, Woszczyk D. Outcome of Ibrutinib Treatment by Baseline Genetic Features in Patients with Relapsed or Refractory CLL/SLL with del17p in the Resonate-17 Study. Blood 2015; 126:493.

77. Sharman JP CS, Furman RR et al. Second interim analysis of a phase 3 study of idelalisib (ZYDELIG) plus rituximab (R) for relapsed chronic lymphocytic leukemia (CLL): efficacy analysis in patient subpopulations with $\mathrm{Del}(17 \mathrm{p})$ and other adverse prognostic factors. Blood 2014;124: 33.

78. Stilgenbauer S, Eichhorst B, Schetelig $J$ et al. Venetoclax in relapsed or refractory chronic lymphocytic leukaemia with $17 \mathrm{p}$ deletion: a multicentre, open-label, phase 2 study. Lancet Oncol 2016;17 (6):768-78.

79. Eichhorst B, Robak T, Montserrat E et al. Chronic lymphocytic leukaemia: ESMO Clinical Practice Guidelines for diagnosis, treatment and follow-up. Ann Oncol 2015; Suppl 5:78-84.

80. Rossi D, Terzi-di-Bergamo L, De Paoli L et al. Molecular prediction of durable remission after first-line fludarabine-cyclophosphamide-rituximab in chronic lymphocytic leukemia. Blood 2015;126(16):1921-4.

81. Fischer K, Bahlo J, Fink AM et al. Long-term remissions after FCR chemoimmunotherapy in previously untreated patients with CLL: updated results of the CLL8 trial. Blood 2016; 127 (2):208-15.

82. Thompson PA, Tam CS, O'Brien SM et al. Fludarabine, cyclophosphamide, and rituximab treatment achieves long-term disease-free survival in IGHV-mutated chronic lymphocytic leukemia. Blood 2016;27(3):303-9. 
83. Herling CD, Klaumünzer M, Krings Rocha $\mathrm{C}$ et al. Complex karyotypes, KRAS and POT1 mutations impact outcome in CLL after chlorambucil based chemo- or chemoimmunotherapy. Blood. 2016 Jul 21;128(3):395-404. doi: 10.1182/blood-2016-01691550. Epub 2016 May 25.

\section{Table 1. Cumulative IIIness Rating Score ${ }^{(31)}$}

\begin{tabular}{|cl|c|}
\hline \multicolumn{2}{|c|}{ Organ-System } & Severity $^{(1)}$ \\
\hline 1. & Cardiac & $0-1-2-3-4$ \\
\hline 2. & Vascular & $0-1-2-3-4$ \\
\hline 3. & Hematological & $0-1-2-3-4$ \\
\hline 4. & Respiratory & $0-1-2-3-4$ \\
\hline 5. & Ophthalmological and ORL & $0-1-2-3-4$ \\
\hline 6. & Upper Gastrointestinal & $0-1-2-3-4$ \\
\hline 7. & Lower Gastrointestinal & $0-1-2-3-4$ \\
\hline 8. & Hepatic and Pancreatic & $0-1-2-3-4$ \\
\hline 9. & Renal & $0-1-2-3-4$ \\
\hline 10. & Genito-urinary & $0-1-2-3-4$ \\
\hline 11. & Muscoloskeletal and cutaneous & $0-1-2-3-4$ \\
\hline 12. & Neurological & $0-1-2-3-4$ \\
\hline 13. & Endocrine, metabolic, mammary & $0-1-2-3-4$ \\
\hline 14. & Psychiatric & $0-1-2-3-4$ \\
\hline
\end{tabular}

(1) Severity score, depending on the extent to which the organ/system is affected: 0, absence of disease 1 ; mild, 2 ; moderate, 3 ; severe; 4 ; very severe. 
Table 2. Granulocytopenia in trials focused on front-line treatment in elderly/unfit patients with CLL

\begin{tabular}{|c|c|c|c|c|}
\hline Author & $\begin{array}{l}\text { Treatment } \\
\text { Schedule }\end{array}$ & No patients & $\begin{array}{l}\text { Median } \\
\text { age }\end{array}$ & $\begin{array}{l}\% \text { grade } \geq 3 \\
\text { neutropenia }\end{array}$ \\
\hline \multirow{2}{*}{$\begin{array}{l}\text { Eichhorst et al. } \\
2015^{(3)}\end{array}$} & FLU & 87 & 71 & \\
\hline & $\mathrm{CHL}$ & 98 & 70 & 12 \\
\hline $\begin{array}{l}\text { Foà et al. } \\
2014^{(18)}\end{array}$ & $\mathrm{CHL}+\mathrm{R}$ & 85 & 70 & \\
\hline $\begin{array}{l}\text { Hillmen et al. } \\
2014^{(17)}\end{array}$ & $\mathrm{CHL}+\mathrm{R}$ & 100 & & 41 \\
\hline $\begin{array}{l}\text { Hillmen et al. } \\
2015^{(20)}\end{array}$ & $\mathrm{CHL}+\mathrm{O}$ & 447 & & 26 \\
\hline $\begin{array}{l}\text { Goede et al. } \\
2014{ }^{(16)}\end{array}$ & $\begin{array}{c}\mathrm{CHL} \\
\mathrm{CHL}+\mathrm{R} \\
\mathrm{CHL}+\mathrm{OBI}\end{array}$ & & 73 & $\begin{array}{l}16 \\
27 \\
35\end{array}$ \\
\hline $\begin{array}{l}\text { O'Brien et al. } \\
2014^{(22)}\end{array}$ & IBR & & 71 & 3.4 \\
\hline $\begin{array}{l}\text { Burger et al. } \\
2015^{(23)}\end{array}$ & $\begin{array}{l}\text { IBR } \\
\mathrm{CHL}\end{array}$ & 99 & 73 & $\begin{array}{l}10 \\
18\end{array}$ \\
\hline $\begin{array}{l}\text { O'Brien et al. } \\
2015^{(25)}\end{array}$ & & 64 & 71 & 28 \\
\hline
\end{tabular}

Abbreviations: TN, treatment naîve; R/R, relapsed/refractory; FLU, fludarabine, $\mathrm{CHL}$, chlorambucil; BR ibrutinib; IDL, idelalisib; R, rituximab;O, ofatumumab; OBI, obinutuzumab; NR, not reported. 
Table 3. 2016 NCNN and 2015 ESMO recommendation for the treatment of aged and/or unfit patients with CLL

\begin{tabular}{|c|c|c|c|}
\hline \multirow{3}{*}{$\begin{array}{l}\text { NCCN } \\
2016 \\
(32)\end{array}$} & \multicolumn{2}{|c|}{ Patients without del (11q) and del (17q)/TP53 mutation ${ }^{(1)}$} & Patients with del(17p)/TP53 mutatio \\
\hline & $\begin{array}{l}>70 \text { years or younger patients } \\
\text { with comorbidities }\end{array}$ & $\begin{array}{l}\text { Frail patients with significant } \\
\text { comorbidities, not able to tolerate } \\
\text { purine analogs }\end{array}$ & All patients \\
\hline & $\begin{array}{ll}\text { In } & \text { order of preference: } \\
\text { - } & \text { Obinutuzumab+Chlorambucil } \\
& \text { (Cat.1) } \\
\text { - } & \text { Ibrutinib (Cat. 1) } \\
\text { - } & \text { Ofatumumab+Chlorambucil } \\
\text { - } & \text { Rituximab+Chlorambucil } \\
\text { - } & \text { Bendamustine } \pm \text { Rituximab } \\
\text { - } & \text { Obinutuzumab (Cat.2B) } \\
\text { - } & \text { Fludarabine } \pm \text { Rituximab (Cat. } \\
& \text { 2B) } \\
\text { - } & \text { Rituximab (Cat.3) }\end{array}$ & $\begin{array}{ll}\text { In } & \text { order of preference: } \\
\text { - } & \text { Obinutuzumab+Chlorambucil } \\
\text { - } & \text { I) } \\
\text { - } & \text { Ofatumumab+Chlorambucil } \\
\text { - } & \text { Rituximab+Chlorambucil } \\
\text { - } & \text { Obinutuzumab (Cat.2B) } \\
\text { - } & \text { Rituximab (Cat.2B) } \\
\text { - } & \text { Chlorambucil (Cat-2B) } \\
\text { - } & \text { Pulse corticosteroids (Cat. 3) }\end{array}$ & $\begin{array}{l}\text { order of preference: } \\
\text { - Ibrutinib } \\
\text { - HDMP+rituximab } \\
\text { - FCR } \\
\text { - FR } \\
\text { - Obinutuzumab+chlorambucil } \\
\text { - Alemtuzumab } \pm \text { rituximab }\end{array}$ \\
\hline \multirow{3}{*}{$\begin{array}{l}\text { ESMO } \\
2015 \\
(79)\end{array}$} & \multicolumn{2}{|c|}{ Patients without del (17p)/TP53 mutation } & $\begin{array}{c}\text { Patients with del (17p)/TP53 } \\
\text { mutation }\end{array}$ \\
\hline & \multicolumn{2}{|c|}{ "Less-fit" } & "Less-fit" \\
\hline & \multicolumn{2}{|c|}{$\mathrm{CHL}+$ Anti-CD20 antibody } & BCR Inhibitor \pm Rituximab \\
\hline
\end{tabular}

\footnotetext{
(1) Category of grading of NCCN recommendations: All recommendations are category $2 \mathrm{~A}$ unless otherwise indicated. 1A: Strong recommendation, high quality evidence; 1B. Strong recommendation, moderate quality evidence; $1 \mathrm{C}$ : Strong recommendation, low quality evidence; $2 \mathrm{~A}$ : Weak recommendation, high quality evidence; $2 \mathrm{~B}$ : Weak recommendation, moderate quality evidence; $2 \mathrm{C}$ : Weak recommendation, low quality evidence.
} 